\section{Control of C. elegans hermaphrodite gonad size and shape by vab-3/Pax6-mediated regulation of integrin receptors}

\author{
Christopher M. Meighan and \\ Jean E. Schwarzbauer ${ }^{1}$ \\ Department of Molecular Biology, Princeton University, \\ Princeton, New Jersey 08544, USA
}

Integrin receptors for extracellular matrix are critical for cell motility, but the signals that determine when to stop are not known. Analysis of distal tip cell (DTC) migration during gonadogenesis in Caenorhabditis elegans has revealed the importance of transcription factor $v a b-3 /$ Pax 6 in regulating the $\alpha$ integrin genes, ina-1 and pat-2. Utilizing vab-3 mutants, we show that the downregulation of ina-1 is necessary for DTC migration cessation and the up-regulation of pat-2 is required for directionality. These results demonstrate concomitant, but distinct roles in migration for each integrin. Notably, transcriptional control of migration termination provides a new mechanism for regulation of morphogenesis and organ size.

Supplemental material is available at http://www.genesdev.org.

Received January 24, 2007; revised version accepted May 21, 2007.

Integrins are heterodimeric transmembrane receptors consisting of $\alpha$ and $\beta$ subunits that link the extracellular matrix (ECM) to the actin cytoskeleton via a wide array of intracellular proteins (Hynes 2002). Through these interactions, integrins regulate cell migration, division, differentiation, and adhesion. Coordinated regulation of integrin adhesive strength over space and time determines the speed and directionality of cell migration in vitro (Palecek et al. 1997; Ridley et al. 2003), and abnormal cell migration has been linked to certain developmental defects caused by loss of integrins in vivo (Bouvard et al. 2001). However, detailed in vivo analyses of integrins in migration are complicated by embryonic lethality of integrin-null mutants and by pleiotropic, redundant, or overlapping functions among the $18 \alpha$ and eight $\beta$ chains in mammals. Some of the experimental constraints inherent in studies on mice can be bypassed by using the nematode Caenorhabditis elegans, which has two conserved integrin receptors composed of an ina $-1 / \alpha$ or pat $-2 / \alpha$ subunit associated with the $p a t-3 / \beta$ integrin subunit (Cox et al. 2004).

Post-embryonic gonadogenesis in C. elegans provides an excellent model of cell migration during development. The shape of the hermaphrodite gonad is dictated

[Keywords: Integrins; cell migration; organogenesis; Pax6] ${ }^{1}$ Corresponding author.

E-MAIL jschwarz@princeton.edu; FAX (609) 258-1035.

Article is online at http://www.genesdev.org/cgi/doi/10.1101/gad.1534807. by migration of two leader cells called the distal tip cells (DTCs) (Hubbard and Greenstein 2000). The DTCs begin to migrate away from the gonad primordium on the ventral ECM of the nematode in larval stage L2 (Fig. 1A). During the third larval stage, they turn and migrate to the dorsal side, followed by a second turn and migration toward the mid-body of the nematode throughout the L4 stage. Migration ends on the dorsal surface approximately opposite the vulva, resulting in two U-shaped gonad arms coincident with the onset of adulthood (Fig. $1 \mathrm{~A}, \mathrm{~B})$. Executing the DTC migratory program requires the coordinated action of matrix metalloproteases, the netrin signaling system, plus integrins and other signaling molecules that regulate the cytoskeleton (Hubbard and Greenstein 2000; Cram et al. 2006). Molecular regulation of gonad size and the signals that stop DTC migration are not known.

The integrin heterodimer composed of INA- $1 / \alpha$ and PAT-3/ $\beta$ is expressed by the DTC throughout migration (Gettner et al. 1995; Baum and Garriga 1997). A weak nonlethal allele of ina-1 causes dorsal migration defects (Baum and Garriga 1997). Similar gonad defects were observed with DTC-specific expression of a dominantnegative pat-3 transgene, with a PAT-3 cytoplasmic tail mutant, or by pat-3 RNA interference (RNAi) (Lee et al. 2001, 2005). Contrary to ina-1, expression of $\alpha$ integrin pat-2 has not been reported in DTCs (http://www. wormbase.org). Lethality of pat-2 mutant alleles due to paralysis and arrest at the twofold stage of embryogenesis occurs prior to gonad morphogenesis (Williams and Waterston 1994).

To further define integrin function during organogenesis and the specific roles of ina-1 and pat-3 during DTC migration, we executed a mutagenesis screen directed toward an ina-1 phenotype. We identified a new allele of $v a b-3$, a gene encoding the ortholog of the transcription factor Pax6. Here we show that the expression of both $\alpha$ integrins is differentially controlled by vab-3/Pax6. Surprisingly, $\alpha$ integrin pat-2 expression is turned on in DTCs during migration and its activity is crucial for dorsal pathfinding. $\alpha$ integrin ina-1, on the other hand, is down-regulated by a vab-3-dependent mechanism, and this effect is necessary for cessation of migration. These results define the functions and regulation of both integrin heterodimers during DTC migration and establish a new morphogenetic mechanism for controlling organ size and shape.

\section{Results and Discussion}

To identify new genes involved in integrin function during DTC migration, nematodes were mutagenized with EMS, then screened for a notched-head phenotype. This defect is observed in certain ina-1 alleles and other mutants that affect cell migration (Baum and Garriga 1997). One mutant, mw105, showed significantly aberrant DTC migration with a highly penetrant migration defect $(99.5 \%, n=400)$ visible as coiled-up gonad arms (Fig. $1 \mathrm{C}, \mathrm{D})$. Ventral migration and DTC turning followed the normal path at the normal rate, but migration was perpetual as it continued throughout the reproductive life span of the nematode. This is in stark contrast to wildtype DTCs, which stop migrating prior to reproduction (Fig. 1B). 

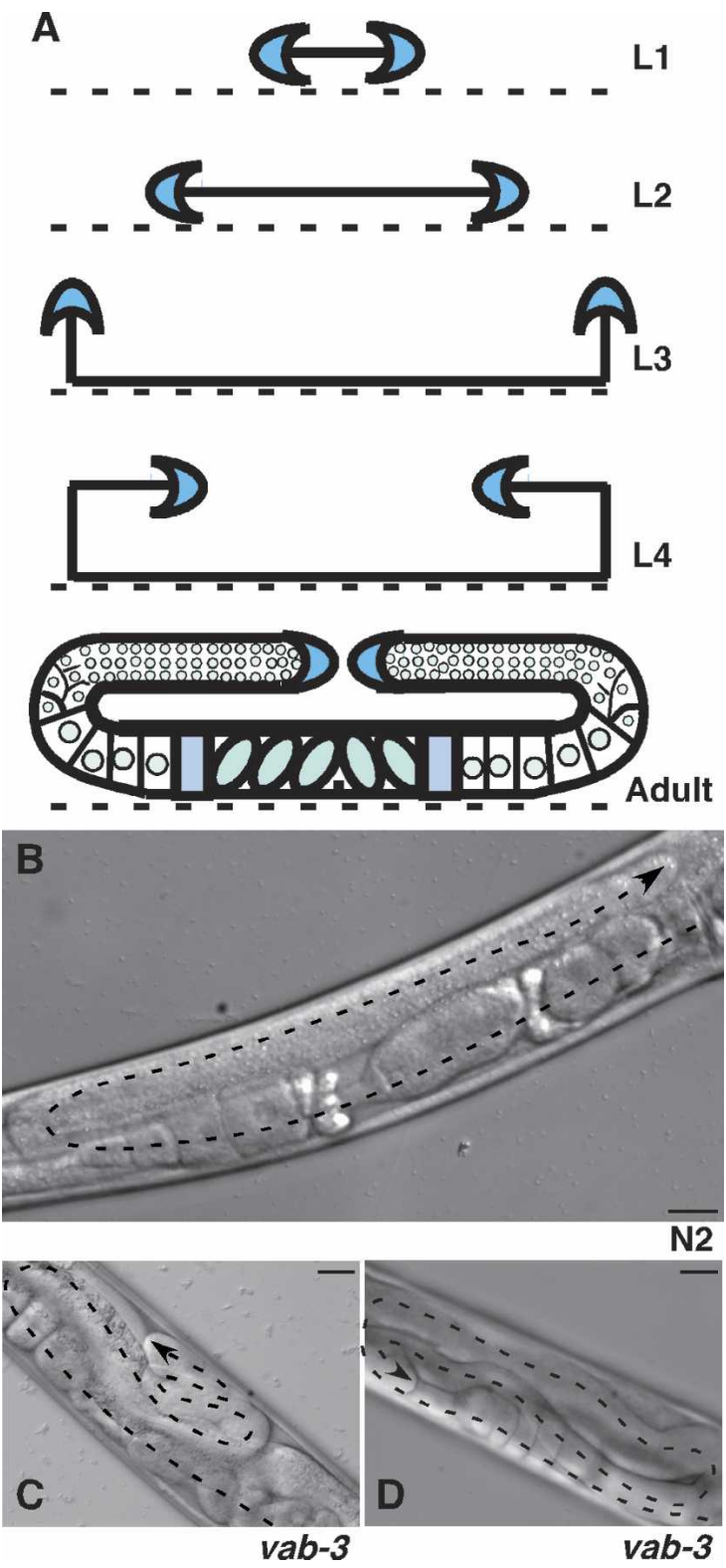

Figure 1. Aberrant DTC migration in vab-3 mutants. (A) DTCs (blue crescents) originate on the ventral surface of the nematode body (dashed line) on the distal edges of the gonad primordium in L1. In L2, they migrate away from the center along the ventral surface, then turn to the dorsal side during L3. A second turn redirects migration along the dorsal surface toward the center of the nematode during L4. Migration then terminates dorsal to the vulva, producing the adult gonad. The developmental stage is indicated at right of each diagram. DIC image of adult wild-type $\mathrm{N} 2$ gonad arm $(B)$, adult $v a b-3(m w 105)$ gonad arm with multiple dorsal turns $(C)$, and $v a b-3(m w 105)$ gonad arm with multiple turns and ventralized DTC migration $(D)$. Dorsal is up, ventral is down. Dashed line traces the gonad arm with arrow at DTC. Bars, $25 \mu \mathrm{m}$.

Mapping experiments localized mw105 to a defined region on the X chromosome. RNAi phenotypes for predicted genes in that region were compared with the mw105 phenotype. Of the 64 RNAi constructs tested, only the clone corresponding to $v a b-3$ yielded a similar DTC migration phenotype. Microinjection of the cosmid F14F3, which contains vab-3 (Chisholm and Horvitz
1995), rescued the mw105 gonad defect, confirming the link between mw105 and vab-3. The vab-3 gene (variable abnormal morphology) encodes a transcription factor homologous to mammalian Pax6 and is defined by the presence of DNA-binding paired and homeodomains (Cinar and Chisholm 2004). DNA sequence analysis revealed a G-to-A transition at the end of exon 2 at base pair 3613, constituting a novel allele that we named $v a b-3(m w 105)$. This missense mutation (G52E) is in a region of the paired domain that makes direct contact with DNA in the crystal structure of Pax6 (Xu et al. 1999). The alteration could abolish interactions with DNA or it could impair, but not completely eliminate VAB-3 binding to target promoters. DTC migration defects have been previously linked to $v a b-3$ mutations; however, the mechanism by which $v a b-3$ causes these defects is unknown (Nishiwaki 1999; Cinar and Chisholm 2004). Phenotypes of vab-3 alleles generally differ based on the location and type of mutation. Nonsense mutations, such as vab-3(e648) with a W101Opal mutation, have frequent notched heads, variably defective DTC migration, and lethality due to larval arrest (Cinar and Chisholm 2004). We find DTC migration defects ranging from a single improper turn to perpetual migration in $65 \%(n=113)$ of $v a b-3(e 648)$ hermaphrodites. In contrast, $v a b-3(e 1796)$ with two missense mutations in the homeodomain (I229T, L232H) gives nematodes with normal heads and no lethality (Cinar and Chisholm 2004), but with the perpetual DTC migration defect $(100 \%, n=54)$. Our mutation $v a b-3(m w 105)$ occurs in the paired domain, yet generates a similar highly penetrant perpetual DTC migration defect to that seen in homeodomain mutant $v a b$ 3(e1796).

In mammals, Pax6 has been linked to transcriptional regulation of genes encoding several adhesion molecules including NCAM, L1CAM, and integrin $\alpha 5$ (Duncan et al. 2000; Simpson and Price 2002). C. elegans $\alpha$ integrin pat-2 is somewhat more homologous to mammalian $\alpha 5$ than to other vertebrate $\alpha$ subunits (Cox et al. 2004). Since pat-2 expression in DTCs has not been reported, we examined DTCs in three independent transgenic lines generated with a transcriptional fusion containing $6 \mathrm{~kb}$ of pat-2 upstream sequence linked to GFP. In each line, the pat-2p::GFP nematodes had high levels of GFP expression in body-wall muscle cells and vulval tissue (see Fig. 2C), in concordance with the expression pattern previously reported (http://www.wormbase.org). No GFP was detected in the DTCs migrating on the ventral ECM during L2 (Fig. 2A-C). In contrast to our expectations, however, GFP was visible in DTCs during L3, when the DTC migrates from the ventral to dorsal surface (Fig. 2D-F), and was maintained in the DTC throughout adulthood (Fig. 2G). Similar DTC expression of GFP was observed in two other independently derived $6-\mathrm{kb}$ pat-2 promoter fusion lines as well as with shorter promoter fusions of 3,2 , and $<0.5 \mathrm{~kb}$ of pat-2 upstream sequence (see below). The pat-2p::GFP strain used in subsequent experiments refers to JE2 121 containing $6 \mathrm{~kb}$ of upstream sequence. To verify that this pattern is an accurate representation of PAT-2 expression, a translational fusion line with GFP inserted near the end of the cytoplasmic tail was examined. The PAT-2::GFP expression pattern was the same as the transcriptional fusions, including the up-regulation of expression in the DTC during L3 (data not shown). 


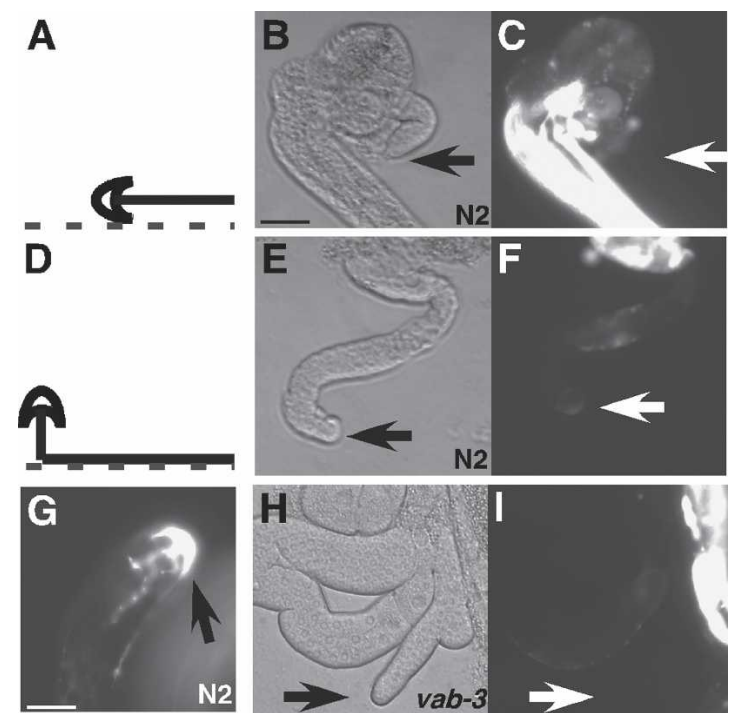

Figure 2. pat-2 is up-regulated in wild-type DTCs, but not in vab-3 mutants. Expression of pat-2 in DTCs was determined using a pat$2 p:: G F P$ transcriptional fusion. Fluorescence was evaluated in dissected gonads to remove masking by bright GFP in body-wall muscles (as in $C)$. $(A-C)$ L2 dissected gonad. The location of DTC at dissection $(A)$ is shown along with DIC $(B)$ and fluorescence $(C)$ images of a representative gonad arm. $(D-F)$ L3 dissected gonad. Diagram of DTC location at dissection $(D)$ with DIC $(E)$ and fluorescence $(F)$ images of dissected gonad arm. $(G)$ Adult dissected gonad with GFP expression in the DTC. $(H, I)$ DIC and fluorescence images of adult dissected gonad from $v a b-3$ mutant crossed into pat-2p::GFP. Arrows point to the DTC. Bars: all panels except $G, 25$ $\mu \mathrm{m} ; G, 12.5 \mu \mathrm{m}$.

Temporal analysis of $v a b-3 p:: G F P$ hermaphrodites showed that GFP expression was turned on in the DTCs during ventral migration, indicating that $\mathrm{VAB}-3$ is present prior to expression of pat-2 (http://www.wormbase.org). To evaluate vab-3 activity on pat-2 expression, $v a b$ 3(e1796) and $v a b-3(m w 105)$ were crossed individually into pat-2p::GFP. GFP up-regulation was compromised on both mutant backgrounds, showing $53 \%(n=111)$ GFPnegative DTCs in vab-3(e1796); pat-2p:: GFP and $45 \%$ $(n=53)$ with vab-3(mw105); pat2p::GFP (Fig. 2H,I). RNAi knockdown of $v a b-3$ on pat-2p::GFP nematodes prevented GFP up-regulation in $70 \%(n=56)$ of DTCs. In each case, the remaining DTCs displayed wild-type pat-2p::GFP expression. The impact of these alleles on pat-2 expression may be incomplete, so a stronger reduction of $v a b-3$ function was generated by $v a b-3$ RNAi on vab-3(e1796); pat-2p::GFP nematodes. This combination prevented up-regulation of GFP in $90 \%(n=48)$ of DTCs (Supplementary Table 1). Therefore, not only is pat-2 expressed by DTCs, but this expression is dependent on $v a b-3$.

Dozier et al. (2001) identified a specific sequence upstream of ceh-32 that interacts with VAB-3. A similar sequence is present 420 base pairs (bp) upstream of pat-2 (Supplementary Fig. 1). We tested the requirement for this sequence by constructing transgenic lines containing wild-type or mutated promoter sequences fused to GFP. The $420 \mathrm{bp}$ of upstream sequence were sufficient to promote DTC expression of GFP $(77 \%, n=60)$. In contrast, alteration of 5 bases in the promoter-GFP construct showed significantly reduced GFP expression $(18 \%, n=56) \quad$ (Supplementary Fig. 1; Supplementary
Table 1). A 381-bp promoter-GFP construct that eliminated this site did not up-regulate GFP $(5 \%, n=44)$. These results strongly suggest that this putative VAB-3binding site is critical for GFP expression driven by the pat-2 promoter, and may be indicative of direct $v a b-3$ regulation of pat-2.

Alleles of pat-2 are lethal prior to gonad formation and RNAi against pat-2 on wild-type N2 nematodes produced many Pat embryos. Some nematodes that were less severely affected by RNAi survived until adulthood with variable defects, including abnormal body size and shape and uncoordinated movement. Those pat-2 RNAi nematodes with normal body size, shape, and movement were evaluated for DTC migration defects. Fifty-three percent had aberrant DTC turns (Fig. 3B), 26\% showed ventralized DTC migration (Fig. 3C), and $21 \%$ had overextension of the DTCs past the vulva $(n=113)$. DTC defects were not seen with RNAi against pat-6, indicating that the pat-2 RNAi effect was dependent on pat-2 knockdown and not on defects in muscle function. Pathfinding defects occurred after entering the L3 stage, and thus after pat-2 expression had been up-regulated. These defects establish a role for pat-2 in determining DTC directionality during dorsal migration and show that loss of pat-2 expression impacts gonad shape. However, they do not completely reproduce the perpetual migration phenotype seen in $v a b-3$ mutants.

The expression pattern of $\alpha$ integrin ina-1 is distinct from pat-2. In wild-type N2 nematodes, ina-1 is expressed in migrating neurons, the pharynx, and the DTCs, but not in body-wall muscles (Baum and Garriga 1997). INA-1::GFP was present in the DTCs at the L2 stage prior to migration and maintained throughout L4
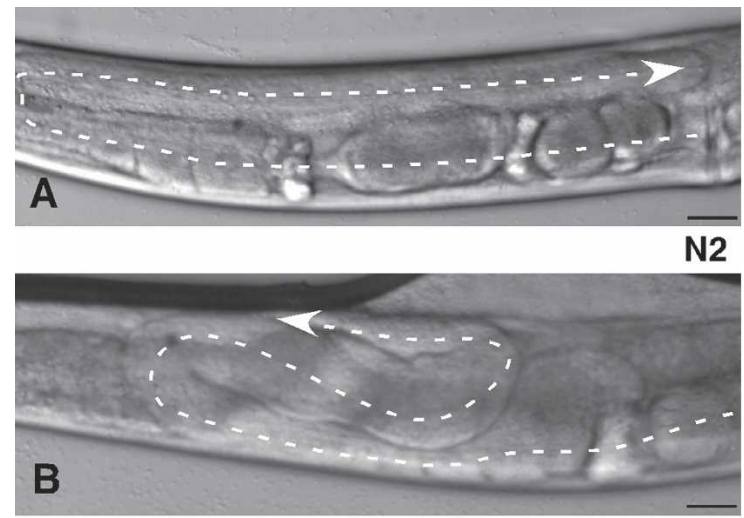

N2, pat-2 RNAi

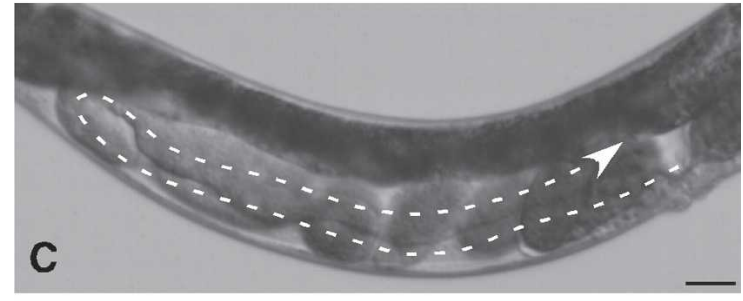

N2, pat-2 RNAi

Figure 3. pat-2 is required for pathfinding during DTC migration. (A) N2 gonad arm has the typical U-shape, as shown in Figure 1B. N2 injected with dsRNA against pat-2 generates an extra turn on the dorsal surface $(B)$ or ventralization of the gonad $(C)$. A dashed line traces the gonad arms, and arrows point to the DTC. Bars, $25 \mu \mathrm{m}$. 
(Fig. 4A). In wild-type N2 adults, INA-1 :: GFP was downregulated with the cessation of migration (2\% GFP-positive, $n=49$ ) (Fig. 4B,D). In contrast to the normal downregulation, INA-1::GFP expression in vab-3(mw105) and $v a b-3(e 1796)$ hermaphrodites was maintained beyond the egg-laying stage $(100 \%, n=63$ and $97 \%, n=38$, respectively) (Fig. 4C,E). Direct comparison of INA$1::$ GFP fluorescence in dissected gonads from agedmatched hermaphrodites showed significantly higher expression in both vab-3 mutants as well as with RNAi against vab-3 than in the wild-type DTC (Fig. 4D,E; Supplementary Table 2). Thus, in a vab-3 mutant with perpetually migrating DTCs, ina-1 expression is also maintained perpetually, showing a strong correlation between ina-1 expression and DTC migration.

$\mathrm{VAB}-3$ is a transcriptional regulator and therefore could control INA-1::GFP expression either through effects on its transcription or by regulating another gene that inactivates the integrin protein. Transgenic nematodes were generated with a transcriptional fusion of the ina-1 promoter with RFP. In these animals, the RFP expression pattern was identical to the INA-1::GFP translational fusion. Expression originated in DTCs prior to migration and was maintained into adulthood. Upon completion of migration, the pattern of RFP in the DTC was reduced to small faint punctate signals (Supplementary Fig. 2). In $100 \%(n=93)$ of ina-1p::RFP nematodes, knock-down of $v a b-3$ by RNAi resulted in RFP maintenance as DTC migration continued throughout egg-laying. Clearly, vab-3 disruption abolishes ina-1 down-regu-
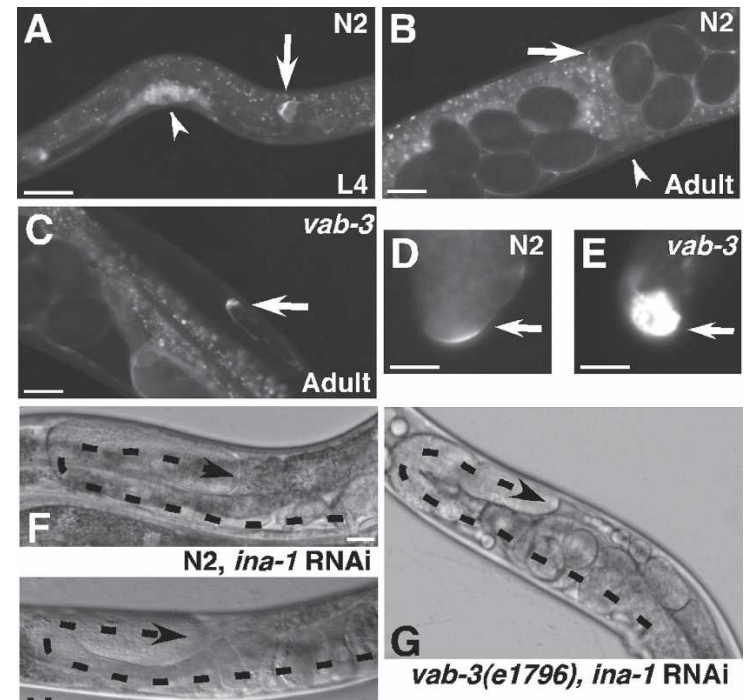

$\mathrm{H}$

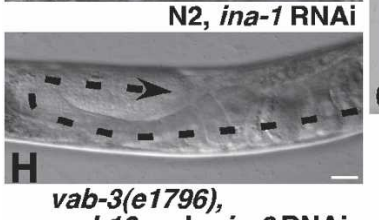

vab-3(e1796), ina-1 RNAi ced-10 and mig-2 RNAi

Figure 4. Ina-1 expression is necessary for normal and perpetual DTC migration. Images of strain NG2517, an INA-1:: GFP translational fusion, show diminishing GFP fluorescence between L4 $(A)$ and adult DTCs $(B) .(C)$ INA-1::GFP is maintained throughout egglaying in the adult $v a b-3$ mutants. $(D, E)$ Gonads were dissected from age-matched NG2517 wild-type or $v a b-3$ mutants expressing INA$1::$ GFP. Fluorescence images were captured at identical settings. Arrows point to DTCs $(A-E)$, and arrowheads point to vulva $(A, B)$. RNAi against ina-1 on N2 $(F)$ or $v a b-3(e 1796)(G)$ nematodes stops DTC migration. Simultaneous RNAi against ced-10 and mig-2 ( $H$ on a vab-3 mutant prevents perpetual migration. Dashed lines trace gonad arms, and arrowheads mark the DTCs. Bars: all panels except $D, E, 25 \mu \mathrm{m} ; D, E, 12.5 \mu \mathrm{m}$. lation at the level of transcription, allowing continuous expression accompanied by perpetual DTC migration. A transcriptional regulator acting to promote the transcription of some genes (pat-2) and prohibit the expression of others (ina-1) in the same cell could itself be regulated by gene-specific cofactors, of which Pax6 is known to have many (Simpson and Price 2002). It is also possible that downstream targets of $v a b-3$ act as transcriptional repressors that then regulate ina-1.

If perpetual DTC migration is dependent on continued ina-1 gene expression, then reduction of INA-1 should rescue the migration defect. Post-embryonic RNAi against ina-1 was carried out on N2 and vab-3(e1796) mutants. Notably, in $66 \%(n=118)$ of ina-1 RNAitreated vab-3(e1796) hermaphrodites, DTCs stopped migrating on the dorsal side with no extra turns. Reduction of ina-1 in N2 nematodes also caused DTC migration to stop $(64 \%, n=80)$ (Fig. 4F,G). Therefore, ina-1 is necessary for DTC migration and extended INA-1 activity is required for the perpetual DTC migration and aberrant gonad size observed in vab-3 mutants. Not only is ina-1 expression important for cell motility, but it also contributes significantly to the regulatory program that controls cessation of migration.

The Rac GTPases CED-10 and MIG-2 have been shown to act downstream from INA-1 during migration of commissural neurons in C. elegans (Poinat et al. 2002). We found that these GTPases are also required for the INA-1-dependent defects in vab-3 mutants. RNAi against ced-10 or mig-2 individually did not reduce the extent of DTC migration in vab-3(e1796) $(0 \%, n=74$ and $0 \%, n=68$, respectively). Knockdown of both GTPases together, however, caused DTC migration to stop $(86 \%, n=42)$ (Fig. $4 \mathrm{H})$. This high percentage of rescue indicates that these GTPases are necessary for perpetual DTC migration, along with the upstream INA-1 receptor, in the vab-3 mutants. Rac-2, on the other hand, is unlikely to be involved, since rac-2 RNAi alone or in combination with knockdown of either ced-10 or mig-2 did not rescue (Supplementary Table 3). Thus, our results identify down-regulation of ina-1 expression as a critical stop signal for controlling DTC migration and gonadogenesis.

The unique, highly penetrant perpetual migration phenotype we have discussed shows that specific stop signs are needed to regulate DTCs at the end of migration. The essential stop signal shown by our results is vab-3-dependent down-regulation of INA-1/ $\alpha$ integrin expression (Fig. 5). Interestingly, pat-2/ $\alpha$ integrin also plays a role in DTC migration, since blockade of its up-regulation midway through gonadogenesis caused pathfinding defects on the dorsal surface. The distinct functional roles for INA-1 in forward movement and PAT-2 in directionality correspond with the opposite effects of $v a b-3$ on expression of these integrins. Control of gonadogenesis by $v a b-3$ and integrin expression provides a way to govern cell migration without changing the ECM environment or modulating presentation of chemoattractants. Simultaneous expression of two integrins could affect migration through differential receptor engagement by their respective ECM ligands, although specific ECM-binding partners have not been determined. Even in the absence of ligands for both integrins, DTC functions could be modulated by receptor cross-talk. For example, transdominant inhibition, in which engagement of one integrin blocks the activity of a second integrin, could result 


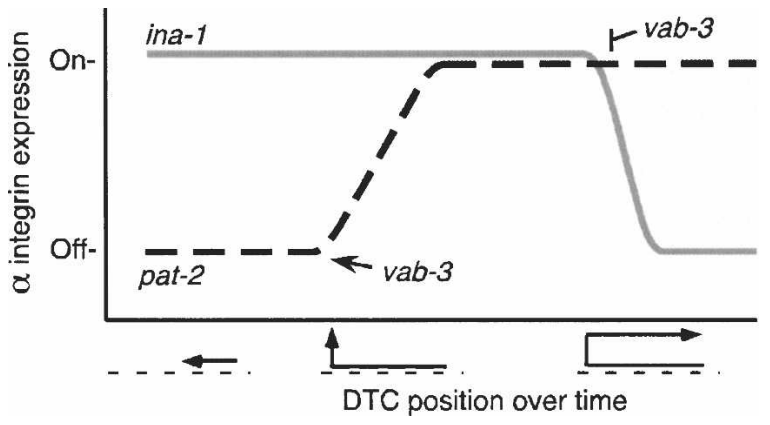

Figure 5. vab-3 modulation of integrin levels during DTC migration. Integrin detection in the DTCs is shown as "Off" or "On" versus a time line of DTC migration progress during gonadogenesis. ina-1 is in gray, and pat-2 is dashed black. As DTC migration begins, ina-1 expression is high and pat-2 is not expressed. vab-3 then upregulates pat-2 during DTC turning. PAT-2 directs dorsal pathfinding and remains on through adulthood. In adults, $v a b-3$ down-regulates ina-1, ending migration.

from up-regulation of PAT-2 reducing INA-1 activity by preferential binding of shared cytosolic factors such as talin (Calderwood et al. 2004). Further definition of the specific activities of each integrin in the context of the other will provide insights into the control of cell-ECM interactions in this and other developmental processes that rely on multiple integrins.

\section{Materials and methods}

Strains and genetics

Nematodes were maintained according to standard protocols (Brenner 1974). N2 was used as wild type. Mutant strains used were CB3304 $v a b-3(e 1796)$ and JE3500 vab-3(mw105). Both strains have very low-penetrance notched-head defects; these defects are more obvious under stress, such as starvation or temperature. Due to poor male generation and male mating problems of $v a b-3$ mutants, all crosses were performed using males generated from the other line involved in the cross. All expression results were identical in both $v a b-3$ alleles. Only $v a b$ 3(e1796) was used for RNAi experiments, as $v a b-3(m w 105)$ is resistant to feeding RNAi. The nature of this resistance is unknown; it could be due to the mutation in $v a b-3$ or the result of a second mutation in a closely linked gene. Transgenic strain NG2517, gmIs5[ina-1::GFP rol-6(su1006)], is a translational fusion of GFP to intact ina-1 including $5 \mathrm{~kb}$ of upstream sequence, able to rescue lethal allele ina-1(gm86) with expression pattern verified by antibody staining (Baum and Garriga 1997). Transgenic strain BC10577 is a transcriptional fusion of $v a b-3$ to GFP (http://www. wormbase.org). Strain JE1111 jeIs1111[ina-1p::RFP rol-6(su1006)] transcriptional fusion (Campbell et al. 2002); JE2121 jeIs2121[pat-2p::GFP rol-6(su1006)] transcriptional fusion, JE2222 jeIs2222[pat-2::GFP rol6(su1006)] translational fusion; JE3521 vab-3(mw105), jeIs2121[pat2p::GFP rol-6(su1006)]; JE3621 vab-3(e1796), jeIs2121[pat-2p::GFP rol6(su1006)]; JE3622 vab-3(e1796), jeIs2222[pat-2::GFP rol-6(su1006)]. Construction of JE1111, JE2121, and JE2222 is described and primer sequences are available in the Supplemental Material.

Mutagenesis, mapping, and sequencing

N2 nematodes were exposed to $47 \mathrm{mM}$ ethyl methanesulfonate according to the standard protocol (Hodgkin 1999). Mutants generated were evaluated with a dissecting microscope for notched heads in the F1 and F2 generations. All notched-head nematodes were evaluated by DIC for gonad defects. Selected mutants were outcrossed with N2 at least twice. Mapping to determine chromosome location utilized strains RW7000 and DH424 in a PCR-based protocol (Williams et al. 1992). Further mapping to specific locations on the X chromosome used strain CB4856 in a PCR, restriction digest-based protocol (Wicks et al. 2001). Sequencing was performed on PCR products from mutant genomic DNA using primers flanking the exons of vab-3 (Chisholm and Horvitz 1995). pat-2 minimal promoter determination

Transcriptional fusions of pat-2 upstream sequences to GFP were constructed as described for strain JE2121. Primers F54A1 and F54BR amplified $6 \mathrm{~kb}$ of pat-2 upstream sequence. GFP was amplified from plasmid pPD95.77 using primers 9577C1F and 9577D1R. These products were used as templates for fusion PCR (Hobert 2002). In individual PCR reactions, forward primers pat $2 \mathrm{p} 3 \mathrm{kbF}$, pat $2 \mathrm{p} 2 \mathrm{kbF}$, pat $2 \mathrm{p} 1 \mathrm{kbF}$, pat $2 \mathrm{p} 420 \mathrm{~F}$, pat2p420mutF, and pat2p381F with reverse primer 9577D2R were used to create GFP fusions with the promoter lengths 3093 bp, 2063 bp, 1005 bp, 420 bp, 420 bp, and 381 bp, and named 3kb, 2kb, 1kb, 420bp, 420bpmut, and $381 \mathrm{bp}$, respectively. Each was individually injected into nematodes; 420bp, 420bpmut and 381bp were gel-purified prior to injection (Jin 1999). These lines carried the transgenes as extrachromosomal arrays. Lines $420 \mathrm{bp}$, 420 mutbp, and $381 \mathrm{bp}$ had neuronal and vulval GFP expression showing that the promoters were still functional and that the loss of GFP expression in the DTCs of 420 mut and 380bp nematodes was due to sequence alterations.

RNAi

Nematodes were exposed to double-stranded RNA (dsRNA) by feeding as described (Kamath et al. 2003). Nematodes were added to plates as eggs, then evaluated for DTC migration as adults to avoid stronger defects that prevented gonad evaluation. The mig-2 RNAi feeding construct contained mig- 2 cDNA from +224 to +475 . This region was amplified by primers mig2F and mig2R, then ligated into pPD129.36 at restriction sites NheI and HindIII. Ced-10 RNAi utilized the entire ced-10 cDNA in feeding vector pPD129.36, a gift from Michael Hurwitz. ina-1, pat-2, rac-2, and pat-6 were obtained from the C. elegans RNAi Library distributed by MRC geneservice, courtesy of Julie Ahringer. The pat-2 construct was used as a template for in vitro transcription to produce dsRNA for injection (Ahringer 2006). All RNAi experiments involving pat-2 were done by injection and only nematodes with normal body sizes were evaluated for DTC migration. The same procedure was followed for pat-6.

\section{Microscopy and dissections}

Fluorescent and DIC images were generated utilizing a Nikon Eclipse TE 2000U microscope equipped for epifluorescence with a Cooke SensiCam High-Performance camera and IP laboratory software (Scanalytics). Nematodes were mounted for viewing as previously described (Cram et al. 2003). To visualize tissues underlying the muscles, gonads were dissected for imaging GFP in DTCs. Dissections were performed in PBS supplemented with $0.2 \mathrm{mM}$ Levamisole. A $26 \mathrm{G}$ needle was used to decapitate the nematodes, followed by evaluation of extruded gonad.

\section{Acknowledgments}

We thank Andy Fire and Roger Tsien for providing plasmids, Maria Martynovsky for the mig-2 RNAi construct, and Gian Garriga, Michael Hurwitz, and the Caenorhabditis Genetics Center, which is funded by the NIH National Center for Research Resources, for strains provided.

\section{References}

Ahringer, J., ed. 2006. Reverse genetics (April 6, 2006), WormBook, ed. The C. elegans Research Community, WormBook, doi/10.1895/ wormbook.1.47.1, http://www.wormbook.org.

Baum, P.D. and Garriga, G. 1997. Neuronal migrations and axon fasciculation are disrupted in ina-1 integrin mutants. Neuron 19: 51-62.

Bouvard, D., Brakebusch, C., Gustafsson, E., Aszodi, A., Bengtsson, T., Berna, A., and Fassler, R. 2001. Functional consequences of integrin gene mutations in mice. Circ. Res. 89: 211-223.

Brenner, S. 1974. The genetics of Caenorhabditis elegans. Genetics 77: 71-94.

Calderwood, D.A., Tai, V., Di Paolo, G., De Camilli, P., and Ginsberg, M.H. 2004. Competition for talin results in trans-dominant inhibition of integrin activation. J. Biol. Chem. 279: 28889-28895.

Campbell, R.E., Tour, O., Palmer, A.E., Steinbach, P.A., Baird, G.S. Zacharias, D.A., and Tsien, R.Y. 2002. A monomeric red fluorescent protein. Proc. Nat1. Acad. Sci. 99: 7877-7882.

Chisholm, A.D. and Horvitz, H.R. 1995. Patterning of the Caenorhabditis elegans head region by the Pax- 6 family member vab-3. Nature 377: 52-55. 
Cinar, H.N. and Chisholm, A.D. 2004. Genetic analysis of the Cae norhabditis elegans pax-6 locus: Roles of paired domain-containing and nonpaired domain-containing isoforms. Genetics 168: 13071322.

Cox, E.A., Tuskey, C., and Hardin, J. 2004. Cell adhesion receptors in $C$. elegans. J. Cell Sci. 117: 1867-1870.

Cram, E.J., Clark, S.G., and Schwarzbauer, J.E. 2003. Talin loss-of-function uncovers roles in cell contractility and migration in C. elegans. J. Cell Sci. 116: 3871-3878.

Cram, E.J., Shang, H., and Schwarzbauer, J.E. 2006. A systematic RNA interference screen reveals a cell migration gene network in C. elegans. J. Cell Sci. 119: 4811-4818.

Dozier, C., Kagoshima, H., Niklaus, G., Cassata, G., and Burglin, T.R. 2001. The Caenorhabditis elegans Six/sine oculis class homeobox gene ceh-32 is required for head morphogenesis. Dev. Biol. 236: 289303.

Duncan, M.K., Kozmik, Z., Cveklova, K., Piatigorsky, J., and Cvekl, A. 2000. Overexpression of PAX6(5a) in lens fiber cells results in cataract and upregulation of $(\alpha) 5(\beta) 1$ integrin expression. J. Cell Sci. 113: 3173-3185.

Gettner, S.N., Kenyon, C., and Reichardt, L.F. 1995. Characterization of $\beta$ pat-3 heterodimers, a family of essential integrin receptors in $C$. elegans. J. Cell Biol. 129: 1127-1141.

Hobert, O. 2002. PCR fusion-based approach to create reporter gene constructs for expression analysis in transgenic C. elegans. Biotechniques 32: 728-730.

Hodgkin, J. 1999. Conventional genetics. In C. elegans: A practical ap proach. (ed. I.A. Hope), pp. 245-270. Oxford University Press, New York.

Hubbard, E.J. and Greenstein, D. 2000. The Caenorhabditis elegans gonad: A test tube for cell and developmental biology. Dev. Dyn. 218 $2-22$.

Hynes, R.O. 2002. Integrins: Bidirectional, allosteric signaling machines. Cell 110: 673-687.

Jin, Y. 1999. Transformation. In C. elegans: A practical approach (ed. I.A. Hope), pp. 69-96. Oxford University Press, New York.

Kamath, R.S., Fraser, A.G., Dong, Y., Poulin, G., Durbin, R., Gotta, M. Kanapin, A., Le Bot, N., Moreno, S., Sohrmann, M., et al. 2003. Systematic functional analysis of the Caenorhabditis elegans genome using RNAi. Nature 421: 231-237.

Lee, M., Cram, E.J., Shen, B., and Schwarzbauer, J.E. 2001. Roles for $\beta$ (pat-3) integrins in development and function of Caenorhabditis elegans muscles and gonads. J. Biol. Chem. 276: 36404-36410.

Lee, M., Shen, B., Schwarzbauer, J.E., Ahn, J., and Kwon, J. 2005. Connections between integrins and Rac GTPase pathways control gonad formation and function in C. elegans. Biochim. Biophys. Acta 1723: 248-255.

Nishiwaki, K. 1999. Mutations affecting symmetrical migration of distal tip cells in Caenorhabditis elegans. Genetics 152: 985-997.

Palecek, S.P., Loftus, J.C., Ginsberg, M.H., Lauffenburger, D.A., and Horwitz, A.F. 1997. Integrin-ligand binding properties govern cell migration speed through cell-substratum adhesiveness. Nature 385: 537540 .

Poinat, P., De Arcangelis, A., Sookhareea, S., Zhu, X., Hedgecock, E.M., Labouesse, M., and Georges-Labouesse, E. 2002. A conserved interaction between $\beta 1$ integrin/PAT-3 and Nck-interacting kinase/MIG15 that mediates commissural axon navigation in C. elegans. Curr. Biol. 12: 622-631.

Ridley, A.J., Schwartz, M.A., Burridge, K., Firtel, R.A., Ginsberg, M.H., Borisy, G., Parsons, J.T., and Horwitz, A.R. 2003. Cell migration: Integrating signals from front to back. Science 302: 1704-1709.

Simpson, T.I. and Price, D.J. 2002. Pax6; a pleiotropic player in development. Bioessays 24: 1041-1051.

Wicks, S.R., Yeh, R.T., Gish, W.R., Waterston, R.H., and Plasterk, R.H 2001. Rapid gene mapping in Caenorhabditis elegans using a high density polymorphism map. Nat. Genet. 28: 160-164.

Williams, B.D. and Waterston, R.H. 1994. Genes critical for muscle development and function in Caenorhabditis elegans identified through lethal mutations. J. Cell Biol. 124: 475-490.

Williams, B.D., Schrank, B., Huynh, C., Shownkeen, R., and Waterston, R.H. 1992. A genetic mapping system in Caenorhabditis elegans based on polymorphic sequence-tagged sites. Genetics 131: 609-624.

Xu, H.E., Rould, M.A., Xu, W., Epstein, J.A., Maas, R.L., and Pabo, C.O.
1999. Crystal structure of the human Pax6 paired domain-DNA complex reveals specific roles for the linker region and carboxy-terminal subdomain in DNA binding. Genes \& Dev. 13: 1263-1275. 


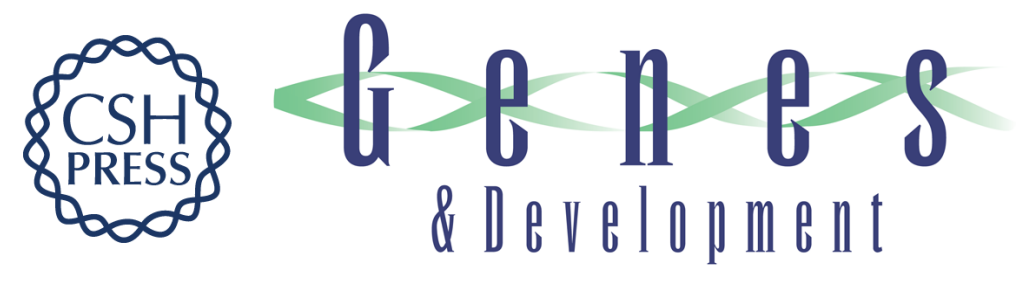

\section{Control of $C$. elegans hermaphrodite gonad size and shape by vab-3 /Pax6-mediated regulation of integrin receptors}

Christopher M. Meighan and Jean E. Schwarzbauer

Genes Dev. 2007, 21:

Access the most recent version at doi:10.1101/gad.1534807

Supplemental http://genesdev.cshlp.org/content/suppl/2007/06/19/21.13.1615.DC1
Material

References This article cites 28 articles, 16 of which can be accessed free at:

http://genesdev.cshlp.org/content/21/13/1615.full.html\#ref-list-1

License

Email Alerting Receive free email alerts when new articles cite this article - sign up in the box at the top

Service

right corner of the article or click here.

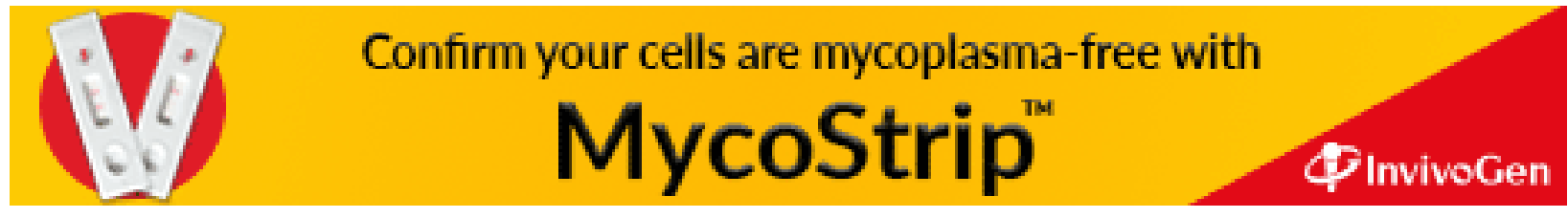

